\title{
Utilization of Sentinel Lymph Node Biopsy in Patients with Ductal Carcinoma In Situ Undergoing Mastectomy
}

\author{
Dhruvil R. Shah, MD, Robert J. Canter, MD, Vijay P. Khatri, MBCHB, Richard J. Bold, MD, \\ Anthony D. Yang, MD, and Steve R. Martinez, MD, MAS \\ Division of Surgical Oncology, Department of Surgery, University of California Davis, \\ Sacramento, CA \\ Steve R. Martinez: steve.martinez@ucdmc.ucdavis.edu
}

\begin{abstract}
Background-Current guidelines suggest consideration of sentinel lymph node biopsy (SLNB) for patients with ductal carcinoma in situ (DCIS) undergoing mastectomy. Our objective was to identify factors influencing the utilization of SLNB in this population.

Methods-We used the Surveillance Epidemiology and End Results database to identify all women with breast DCIS treated with mastectomy from 2000 to 2008. We excluded patients without histologic confirmation, those diagnosed at autopsy, those who had axillary lymph node dissections performed without a preceding SLNB, and those for whom the status of SLNB was unknown. We used multivariate logistic regression reporting odds ratios (OR) and $95 \%$ confidence intervals (CI) to evaluate the relationship of patient- and tumor-related factors to the likelihood of undergoing SLNB.
\end{abstract}

Results-Of 20,177 patients, $51 \%$ did not receive SLNB. Factors associated with a decreased likelihood of receiving a SLNB included advancing age (OR 0.66; $95 \%$ CI 0.62-0.71), Asian (OR 0.75 ; CI $0.68-0.83$ ) or Hispanic (OR $0.84 ; 95 \%$ CI 0.74-0.96) race/ethnicity, and history of prior non-breast (OR 0.57; $95 \%$ CI 0.53-0.61). Factors associated with an increased likelihood of receiving a SLNB included treatment in the east (OR 1.28; $95 \%$ CI 1.17-1.4), intermediate (OR $1.25 ; 95 \%$ CI 1.11-1.41), high (OR 1.84; $95 \%$ CI 1.62-2.08) grade tumors, treatment after the year 2000, and DCIS size 2-5 cm (OR 1.54; $95 \%$ CI 1.42-1.68) and $>5 \mathrm{~cm}$ (OR 2.43; $95 \% \mathrm{CI}$ 2.16-2.75).

Conclusions-SLNB is increasingly utilized in patients undergoing mastectomy for DCIS, but disparities in usage remain. Efforts at improving rates of SLNB in this population are warranted.

Increasing breast cancer awareness and screening mammography have resulted in an increase in the incidence of ductal carcinoma in situ (DCIS). ${ }^{1}$ Whereas both breast conservation therapy and mastectomy are options for the surgical treatment of DCIS, there is recent trend among patients favoring mastectomy. ${ }^{2,3}$ Sentinel lymph node biopsy (SLNB) is

Presented in part at as an oral presentation at the 65th Annual Cancer Symposium of the Society of Surgical Oncology, Orlando, FL, March 21-24, 2012.

Electronic supplementary material The online version of this article (doi:10.1245/s10434-012-2539-4) contains supplementary material, which is available to authorized users. 
an established, minimally invasive method of lymph node assessment in women with clinically node-negative breast cancer who undergo mastectomy. By definition, DCIS lacks an invasive component and therefore has no potential to metastasize to lymph nodes or elsewhere. However, approximately $10-38 \%$ of patients diagnosed with DCIS on coreneedle biopsy harbor occult invasive cancer that is only detected on final pathologic examination. ${ }^{4-8}$ Past and current guidelines suggest consideration of SLNB in patients undergoing mastectomy for DCIS (level 2A evidence). ${ }^{9,10}$ The rationale for this is based on the observation that mastectomy disrupts breast lymphatic drainage patterns enough to preclude SLNB after mastectomy should invasive cancer be incidentally found on final pathology. Our objective was to determine how often SLNB is used in women undergoing mastectomy for DCIS and to identify patient, tumor, and geographic factors that influence utilization of SLNB in these women.

\section{METHODS}

We used the Surveillance, Epidemiology, and End Results (SEER) database to identify all woman treated by mastectomy for DCIS of the breast diagnosed from 2000 through 2008. All cases represent patients with pure DCIS diagnosed on final pathology. SEER collects cancer incidence, prevalence, and survival data from 17 population-based cancer registries representing $28 \%$ of the U.S. population. SEER furthermore provides information on patient demographics, primary tumor site, tumor morphology, stage at diagnosis, and first course of treatment. The population reporting to SEER is comparable to the general population with respect to measures of poverty and education but is slightly more urban than the general U.S. population. Current SEER registries consist of the states of Connecticut, Hawaii, Iowa, Kentucky, Louisiana, New Jersey, New Mexico, and Utah; the metropolitan areas of Atlanta, Detroit, San Francisco-Oakland, Seattle-Puget Sound, and San JoseMonterey; and the Alaska Native Tumor Registry, rural Georgia, Greater California, and Los Angeles County.

Patients were identified as having had a SLNB only if this was specifically coded. All tumor size measurements reported represent an in situ component only; patients with invasive breast cancer, synchronous DCIS, and prior breast malignancy were excluded. We also excluded patients without histologic confirmation, those diagnosed at autopsy, those who had axillary lymph node dissections (ALND) performed for axillary staging without a preceding SLNB, and those for whom the status of SLNB was unknown.

Multivariate logistic regression evaluated the relationship between tumor- and patientrelated factors on the likelihood of undergoing SLNB. Covariates evaluated included patient age ( smean age or >mean age), race/ ethnicity (white, black, Asian, Hispanic, Native American, unknown), year of diagnosis (2000-2008), tumor grade (low, intermediate, high, unknown), size of DCIS ( $<2,2-5,>5 \mathrm{~cm}$, unknown), estrogen receptor (ER) status (positive, negative, borderline, unknown), and regional location of SEER registry (west, midwest, northeast, south). We determined whether patients' DCIS represented their only known malignancy or if they had additional non-breast cancers before their diagnosis of DCIS. An additional multivariate model was constructed that differed only in its inclusion of patients for whom DCIS was their only reported malignancy. To account for the impact of recent 
guidelines on disparities in utilization for SLNB, a third multivariate model was constructed including only patients diagnosed between 2006 and 2008. All models included women with incomplete data regarding size, grade, ER status, and race, because these predictors may not necessarily be available to all surgeons at the time of surgery for DCIS.

We used odds ratios (OR) and $95 \%$ confidence intervals (CI) to identify significant associations. All statistical analyses were done using STAT 11 (College Station, TX) and R version 2.14.1 (http://www.R-project.org). Because information in SEER contains deidentified patient data, this study was exempt from institutional review board approval.

\section{RESULTS}

A total of 20,177 woman met inclusion criteria. Table 1 depicts the patient- and tumorrelated characteristics of the study cohort.

The mean age was 56 years, and the majority was white women $(75 \%)$ who resided in states incorporated into western SEER registries $(58 \%)$. The majority of patients had no prior history of malignancy ( $73 \%$ ), whereas $27 \%$ of patients had a non-breast malignancy before the diagnosis of DCIS. Overall, $51 \%$ of patients did not receive SLNB at the time of mastectomy. By contrast, of those for whom DCIS was their first or only primary tumor, 46 $\%$ did not receive SLNB. Among those who received a SLNB, 13 and $29 \%$ had incomplete data regarding grade and size focus of DCIS, respectively. Among women with known DCIS grade, SLNB was performed more often for high-grade DCIS (58 \%) followed by intermediate-grade (47\%), and low-grade (38\%; Fig. 1a). Similarly, when stratified by DCIS size, a greater proportion of women with DCIS $>5 \mathrm{~cm}(71 \%)$ received a SLNB than women with DCIS between 2 and $5 \mathrm{~cm}(58 \%)$ and women with DCIS that was $<2 \mathrm{~cm}$ (45 $\%$; Fig. 1b). Hormone receptor status was not assessed or was unknown in $57-60 \%$ of patients.

The proportion of SLNBs performed significantly increased with time disproportionate to the increase in the use of mastectomy for DCIS (Fig. 2). Use of SLNB increased from $19 \%$ of all mastectomies in the year 2000 to $70 \%$ of all mastectomies in the year 2008 .

Table 2 depicts the results of the multivariate logistic regression model assessing the odds of undergoing SLNB for the entire patient cohort. Factors associated with increased odds of SLNB included: year of diagnosis, increasing DCIS grade and size, and eastern regional location. Factors associated with a decreased likelihood of receiving a SLNB included age older than 56 years, Asian or Hispanic race, and history of prior non-breast malignancy. We further looked at patterns of SLNB among patients without a prior history of malignancy ( $N$ $=14,699)$. Our findings in this limited model were unchanged, except that Hispanic ethnicity was no longer a significant predictor of receiving SLNB (Table 3). Finally, we examined significant predictors of SLNB in a more contemporary model of patients who underwent mastectomy for DCIS between 2006-2008 ( $N=7,883)$. In this model, increasing DCIS grade and size, but not geographic location, continued to be associated with SLNB in DCIS Patients Undergoing Mastectomy 25 increased odds of undergoing SLNB, whereas 
patients older than 56 years, of Asian ethnicity, or with a history of prior malignancy remained less likely to receive SLNB.

\section{DISCUSSION}

Those who oppose SLNB at the time of mastectomy for DCIS cite the low rate (1-10 \%) of lymph node metastasis in DCIS diagnosed by core needle biopsy, even among patients with high risk DCIS. ${ }^{1,11-13}$ However, the clinical significance of lymph node metastases in DCIS patients is unclear, because majority of these metastases are isolated tumor cells. Among 470 patients with DCIS undergoing SLNB, Moore et al. noted 47 cases $(9 \%)$ of metastasis. ${ }^{14}$ However, true upstaging occurred in only $1.5 \%$ of patients if all cases of isolated tumor were excluded. Moreover, El-Tamer et al. demonstrated no difference in overall survival and disease-specific survival in patients with pure DCIS with only isolated tumor cells or micrometastases found on axillary lymph node dissection. ${ }^{15}$

Proponents of SLNB at the time of mastectomy argue that there is no other way to stage the lymph node basin reliably. If invasive breast cancer is identified after mastectomy, an SLNB can no longer be performed reliably due to altered lymphatic drainage of the breast. ${ }^{16}$ One must then decide between observation, which may lead to understaging and therefore undertreatment, or ALND, which is associated with significantly higher rates of morbidity than SLNB. ${ }^{17}$ Studies have documented upstaging to invasive breast carcinoma in approximately $10-38 \%$ of patients diagnosed with DCIS on core-needle biopsy. ${ }^{4-8}$

These studies prompted a major change to the 2008 NCCN guidelines, which previously did not recommend SLNB in patients undergoing mastectomy for DCIS. However, American Society of Clinical Oncology (ASCO) guidelines have recommend consideration of SLNB in these patients since 2005. The difference in timing of these recommendations may account for the low (51\%) overall observed rate of SLNB in women undergoing mastectomy as well as for the observed disparities in use associated with tumor-related (grade and size of DCIS focus) and non-tumor- related factors (age, year of diagnosis, race/ ethnicity, and geographic location). These disparities reflect the evolving understanding of the pathology of DCIS and the somewhat controversial nature of lymph node assessment in patients with DCIS.

Both high grade and large DCIS size have been associated with increased risk of harboring invasive cancer, which may explain why patients with these characteristics were more likely to undergo SLNB at time of mastectomy in our study. ${ }^{7,18}$ Risk of invasive cancer also is increased with DCIS recurrence, which may explain why women without a prior primary tumor were less likely to undergo SLNB than women with a history of prior cancers.

Age-related, racial, and geographic disparities have been previously documented in women with invasive cancer undergoing SLNB. A study by Reeder-Hayes et al. ${ }^{19-22}$ using SEER to evaluate women with early-stage breast cancer found that black women and those older than age 80 years were less likely to undergo SLNB. Other studies have documented similar results. Interestingly, although we did observe similar age-related disparities in the use of SLNB, there was not such a disparity in blacks. Rather, a lower likelihood of SLNB was 
noted among Asian women. This may reflect the relatively larger growth in underserved Asian population. According to the 2010 United States Census, the Asian population increased by $43 \%$ since the year 2000, whereas the African American population increased by only $12 \%$.Most of this growth was in the south and west, where the population increased by $13-15 \%$ during the past decade compared with a $3 \%$ increase in the northeast. This shifting in census may explain the geographic disparities that we observed and why they vary depending on the years studied. ${ }^{20}$

A previous SEER analysis performed between 1998 and 2002 demonstrated that nearly 87 $\%$ of women who had mastectomy and lymph node assessment for DCIS had ALND, whereas only $13 \%$ underwent SLNB. ${ }^{23}$ Despite the low overall rate of SLNB in our study, we did see an increasing trend in its utilization, which was disproportionate to the overall increase in mastectomies during the past 5 years. This perhaps reflects the increasing acceptance of SLNB following the release of the 2005 ASCO guidelines advocating its use and continued divergence from the use of ALND.

Our study has several limitations inherent to the utilization of population-based data. Our patient population was limited because we excluded cases of DCIS before the year 2000. We chose these years because SEER did not start coding for SLNB until 1998, and we wanted to avoid early irregularities in coding that might have existed. Our data also could be subject to selection bias. Our patient population includes only patients with a diagnosis of DCIS on their final histopathology. Ideally, we would like to capture the entire cohort of patients who had DCIS diagnosed on initial core biopsy, including those that were subsequently diagnosed with invasive carcinoma at mastectomy. Our overall rate of utilization of SLNB might be higher if such patients were included in our cohort. Data completeness also could have influenced our data. SEER does not report information on patient preference, prior surgical or pathologic information, and pregnancy status, which may influence surgical decision making. This is especially important in the $27 \%$ of women with additional nonbreast tumor diagnoses. It is possible that these women were deemed higher risk for invasive malignancy based on previous treatment, such as chest wall radiation, which could influence use of SLNB. We included these women in our analysis, because SLNB would still be indicated in these women using current guidelines.

If trends continue and more women undergo mastectomy for DCIS, it is going to be important to identify disparities in care consistent with national consensus guidelines. This will allow specifically targeted patient education, physician training, and further research in these areas. We have demonstrated that although utilization of SLNB is increasing, it remained underutilized in older women of racial/ethnic minorities and those with low-risk DCIS. These potential factors that promote underutilization of SLNB are areas that can be addressed by further research or educational programs.

\section{Supplementary Material}

Refer to Web version on PubMed Central for supplementary material. 


\section{Acknowledgments}

Supported by Grant Number UL1 RR024146 from the National Center for Research Resources (NCRR) a component of the National Institutes of Health (NIH), and NIH Roadmap for Medical Research. The content is solely the responsibility of the authors and does not necessarily represent the official views of the NNCRR or NIH. Information on NCRR is available at http://www.ncrr.nih.gov/. Information on Re-engineering the Clinical Research Enterprise can be obtained from http://nihroadmap.nih.gov/clinicalresearch/overview-translational.asp.

\section{References}

1. Virnig BA, Tuttle TM, Shamliyan T, Kane RL. Ductal carcinoma in situ of the breast: a systematic review of incidence, treatment, and outcomes. J Natl Cancer Inst. 2010; 102:170-8. [PubMed: 20071685]

2. Yao K, Stewart AK, Winchester DJ, Winchester DP. Trends in contralateral prophylactic mastectomy for unilateral cancer: a report from the National Cancer Data Base, 1998-2007. Ann Surg Oncol. 2010; 17:2554-62. [PubMed: 20461470]

3. McGuire KP, Santillan AA, Kaur P, et al. Are mastectomies on the rise? A 13-year trend analysis of the selection of mastectomy versus breast conservation therapy in 5865 patients. Ann Surg Oncol. 2009; 16:2682-90. SLNB in DCIS Patients Undergoing Mastectomy 29. [PubMed: 19653046]

4. Mittendorf EA, Arciero CA, Gutchell V, et al. Core biopsy diagnosis of ductal carcinoma in situ: an indication for sentinel lymph node biopsy. Curr Surg. 2005; 62:253-7. [PubMed: 15796952]

5. Wilkie C, White L, Dupont E, et al. An update of sentinel lymph node mapping in patients with ductal carcinoma in situ. Am J Surg. 2005; 190:563-6. [PubMed: 16164920]

6. Intra M, Rotmensz N, Veronesi P, et al. Sentinel node biopsy is not a standard procedure in ductal carcinoma in situ of the breast: the experience of the European Institute of Oncology on 854 patients in 10 years. Ann Surg. 2008; 247:315-9. [PubMed: 18216539]

7. Yen TW, Hunt KK, Ross MI, et al. Predictors of invasive breast cancer in patients with an initial diagnosis of ductal carcinoma in situ: a guide to selective use of sentinel lymph node biopsy in management of ductal carcinoma in situ. J Am Coll Surg. 2005; 200:516-26. [PubMed: 15804465]

8. Dillon MF, McDermott EW, Quinn CM, et al. Predictors of invasive disease in breast cancer when core biopsy demonstrates DCIS only. J Surg Oncol. 2006; 93:559-63. [PubMed: 16705731]

9. Lyman GH, Giuliano AE, Somerfield MR, et al. American Society of Clinical Oncology guideline recommendations for sentinel lymph node biopsy in early-stage breast cancer. J Clin Oncol. 2005; 23:7703-20. [PubMed: 16157938]

10. ACR-ACS-CAP-SSO Practice Guideline for the Management of Ductal Carcinoma In-Situ of the Breast. 2006. http://www.acr.org/SecondaryMainMenuCategories/quality_safety/guidelines/breast/ DCIS.pdf

11. Sakr R, Barranger E, Antoine M, et al. Ductal carcinoma in situ: value of sentinel lymph node biopsy. J Surg Oncol. 2006; 94:426-30. [PubMed: 16967457]

12. Katz A, Gage I, Evans S, et al. Sentinel lymph node positivity of patients with ductal carcinoma in situ or microinvasive breast cancer. Am J Surg. 2006; 191:761-6. [PubMed: 16720145]

13. Klauber-DeMore N, Tan LK, Liberman L, et al. Sentinel lymph node biopsy: is it indicated in patients with high-risk ductal carcinoma in situ and ductal carcinoma-in-situ with microinvasion? Ann Surg Oncol. 2000; 7:636-42. [PubMed: 11034239]

14. Moore KH, Sweeney KJ, Wilson ME, et al. Outcomes for women with ductal carcinoma-in-situ and a positive sentinel node: a multi-institutional audit. Ann Surg Oncol. 2007; 14:2911-7. [PubMed: 17597346]

15. El-Tamer M, Chun J, Gill M, et al. Incidence and clinical significance of lymph node metastasis detected by cytokeratin immunohistochemical staining in ductal carcinoma in situ. Ann Surg Oncol. 2005; 12:254-9. [PubMed: 15827818]

16. Newman EA, Cimmino VM, Sabel MS, et al. Lymphatic mapping and sentinel lymph node biopsy for patients with local recurrence after breast-conservation therapy. Ann Surg Oncol. 2006; 13:527. [PubMed: 16372155] 
17. Crane-Okada R, Wascher RA, Elashoff D, Giuliano AE. Long-term morbidity of sentinel node biopsy versus complete axillary dissection for unilateral breast cancer. Ann Surg Oncol. 2008; 15:1996-2005. [PubMed: 18415650]

18. Tan JC, McCready DR, Easson AM, Leong WL. Role of sentinel lymph node biopsy in ductal carcinoma-in-situ treated by mastectomy. Ann Surg Oncol. 2007; 14:638-45. [PubMed: 17103256]

19. Reeder-Hayes KE, Bainbridge J, Meyer AM, et al. Race and age disparities in receipt of sentinel lymph node biopsy for early-stage breast cancer. Breast Cancer Res Treat. 2011; 128:863-71. [PubMed: 21340480]

20. Chen AY, Halpern MT, Schrag NM, et al. Disparities and trends in sentinel lymph node biopsy among early-stage breast cancer patients (1998-2005). J Natl Cancer Inst. 2008; 100:462-74. [PubMed: 18364506]

21. Halpern MT, Chen AY, Marlow NS, Ward E. Disparities in receipt of lymph node biopsy among early-stage female breast cancer patients. Ann Surg Oncol. 2009; 16:562-70. [PubMed: 18998064]

22. Baxter NN, Virnig BA, Durham SB, Tuttle TM. Trends in the treatment of ductal carcinoma in situ of the breast. J Natl Cancer Inst. 2004; 96:443-8. [PubMed: 15026469]

23. Porembka MR, Abraham RL, Sefko JA, et al. Factors associated with lymph node assessment in ductal carcinoma in situ: analysis of 1988-2002 seer data. Ann Surg Oncol. 2008; 15:2709-19. [PubMed: 18483831] 
a

Percent

80

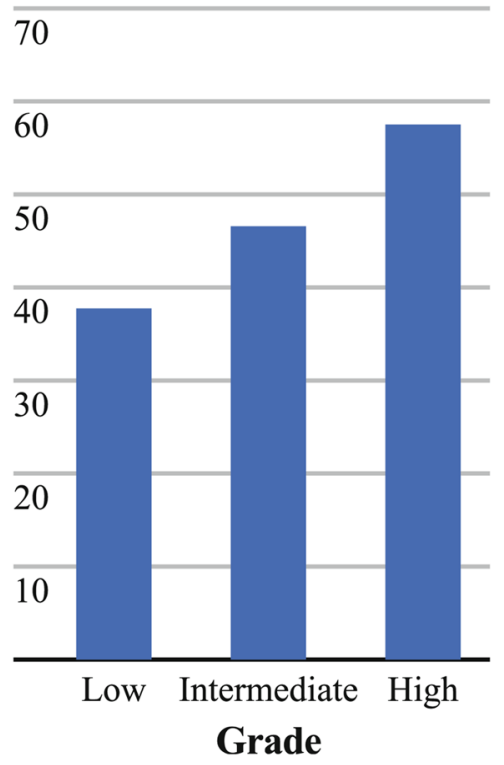

b

Percent

80

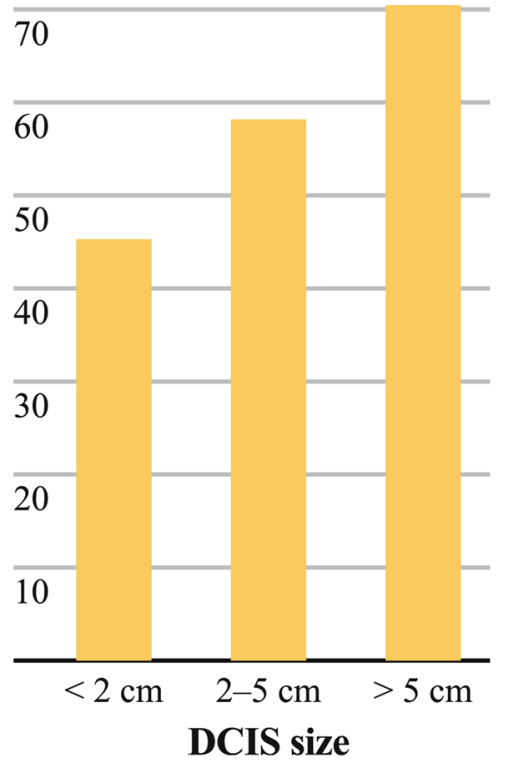

FIG. 1.

a Proportion of sentinel lymph nodes biopsies performed by DCIS grade. b Proportion of sentinel lymph node biopsies performed by DCIS size 
$\%$ of all mastectomies $(\mathrm{n}=20177)$

Percent

$\%$ SLN biopsy per mastectomy

80

70

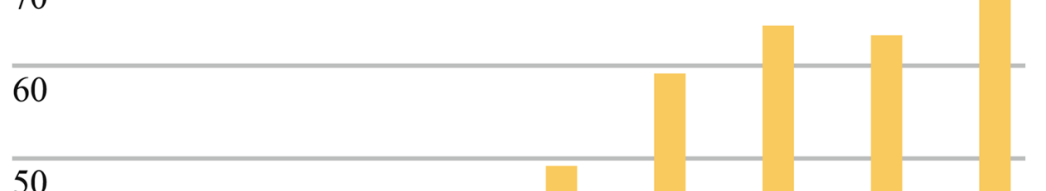

50

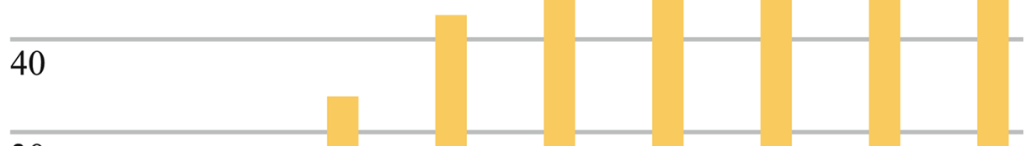

30

20

10

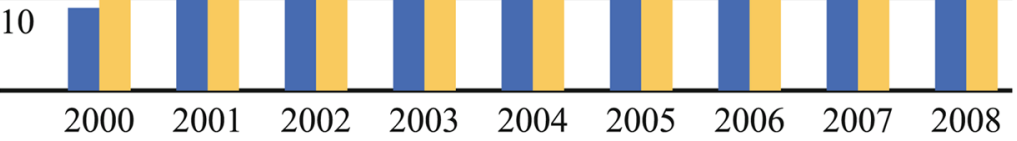

FIG. 2.

Percentage of all mastectomies performed for the entire cohort in a given year (blue bars) as well as percentage of women who received sentinel lymph node biopsy per total mastectomies performed in a given year (gold bars) 
TABLE 1

Patient and tumor characteristics of 20,177 women undergoing mastectomy for DCIS

\begin{tabular}{|c|c|c|c|c|}
\hline & Total $N=20,177(\%)$ & SLNB $N=10,026(\%)$ & No SLNB $N=10,151(\%)$ & $P$ \\
\hline Age (years) & & & & $<0.01$ \\
\hline$<40$ & $1,116(6)$ & $676(7)$ & $440(4)$ & \\
\hline $40-49$ & $5,088(25)$ & $2,879(29)$ & $2,209(22)$ & \\
\hline $50-59$ & $5,589(28)$ & $2,883(29)$ & $2,706(27)$ & \\
\hline $60-69$ & $4,221(21)$ & $2,029(20)$ & $2,192(22)$ & \\
\hline $70-79$ & $3,031(15)$ & $1,211(12)$ & $1,820(18)$ & \\
\hline $80+$ & $1,132(6)$ & $348(4)$ & $784(8)$ & \\
\hline SEER registry & & & & $<0.01$ \\
\hline West & $11,743(58)$ & $5,888(59)$ & $5,855(58)$ & \\
\hline East & $3,298(16)$ & $1,693(17)$ & $1,605(16)$ & \\
\hline Midwest & $3,343(17)$ & $1,590(16)$ & $1,753(17)$ & \\
\hline South & $1,793(9)$ & $855(9)$ & $938(9)$ & \\
\hline Race & & & & 0.35 \\
\hline White & $14,989(75)$ & $7,475(75)$ & $7,514(74)$ & \\
\hline Asian or PI & $2,129(11)$ & $1,010(10)$ & $1,119(11)$ & \\
\hline Black & $1,644(8)$ & $821(8)$ & $823(8)$ & \\
\hline Hispanic & $1,222(6)$ & $620(6)$ & $602(6)$ & \\
\hline Native American/ & $84(<1)$ & $45(<1)$ & $39(<1)$ & \\
\hline Alaskan & & & & \\
\hline Unknown & $109(1)$ & $55(1)$ & $54(1)$ & \\
\hline Grade & & & & $<0.01$ \\
\hline Low & $1,685(8)$ & $638(6)$ & $1,047(10)$ & \\
\hline Intermediate & $6,009(30)$ & $2,797(28)$ & $3,212(32)$ & \\
\hline High & $9,133(45)$ & $5,254(52)$ & $4,329(48)$ & \\
\hline Unknown & $3,350(17)$ & $1,337(13)$ & $2,013(20)$ & \\
\hline Size $(\mathrm{cm})$ & & & & $<0.01$ \\
\hline$<2$ & $7,843(39)$ & $3,565(36)$ & $4,278(42)$ & \\
\hline $2-5$ & $3,996(20)$ & $2,326(23)$ & $1,670(16)$ & \\
\hline$>5$ & $1,731(9)$ & $1,223(12)$ & $508(5)$ & \\
\hline Unknown & $6,607(33)$ & $2,912(29)$ & $3,695(36)$ & \\
\hline ER & & & & $<0.01$ \\
\hline Positive & $6,687(33)$ & $4,413(44)$ & $2,274(22)$ & \\
\hline Equivocal & $27(<1)$ & $20(<1)$ & $7(<1)$ & \\
\hline Negative & $1,967(10)$ & $1,423(14)$ & $544(5)$ & \\
\hline Unknown & $11,496(57)$ & $4,170(42)$ & $7,326(72)$ & \\
\hline Prior non-breast malignancy & & & & $<0.01$ \\
\hline No & $14,698(73)$ & $7,881(79)$ & $6,817(67)$ & \\
\hline Yes & $5479(27)$ & $2,145(21)$ & $3,334(33)$ & \\
\hline
\end{tabular}

$S L N B$ sentinel lymph node biopsy, ER estrogen receptor, DCIS ductal carcinoma in situ 


\section{TABLE 2}

Patient and tumor characteristics associated with the odds of receiving a sentinel lymph node biopsy in entire cohort $(N=20,177)$

\begin{tabular}{|c|c|c|c|}
\hline & OR & $95 \% \mathrm{CI}$ & $P$ \\
\hline \multicolumn{4}{|l|}{ Age (years) } \\
\hline$\leq 56$ & Reference & & \\
\hline$>56$ & 0.66 & $0.62-0.71$ & $<0.01$ \\
\hline \multicolumn{4}{|l|}{ Year } \\
\hline 2000 & Reference & & \\
\hline 2001 & 1.52 & $1.3-1.78$ & $<0.01$ \\
\hline 2002 & 2.09 & $1.8-2.43$ & $<0.01$ \\
\hline 2003 & 3.04 & $2.62-3.53$ & $<0.01$ \\
\hline 2004 & 2.92 & $2.5-3.41$ & $<0.01$ \\
\hline 2005 & 4.08 & $3.48-4.79$ & $<0.01$ \\
\hline 2006 & 5.17 & $4.41-6.08$ & $<0.01$ \\
\hline 2007 & 5.04 & $4.29-5.91$ & $<0.01$ \\
\hline 2008 & 6.7 & $5.71-7.88$ & $<0.01$ \\
\hline \multicolumn{4}{|l|}{ SEER registry } \\
\hline West & Reference & & \\
\hline East & 1.28 & $1.17-1.4$ & $<0.01$ \\
\hline Midwest & 0.92 & $0.84-1.00$ & 0.77 \\
\hline South & 1.03 & $0.92-1.16$ & 0.33 \\
\hline \multicolumn{4}{|l|}{ Race } \\
\hline White & Reference & & \\
\hline Asian or PI & 0.75 & $0.68-0.83$ & $<0.01$ \\
\hline Black & 0.91 & $0.81-1.02$ & 0.18 \\
\hline Hispanic & 0.84 & $0.74-0.96$ & 0.03 \\
\hline Native American/Alaskan & 1.02 & $0.64-1.64$ & 0.99 \\
\hline Unknown & 0.78 & $0.52-1.17$ & 0.34 \\
\hline \multicolumn{4}{|l|}{ Grade } \\
\hline Low & Reference & & \\
\hline Intermediate & 1.25 & $1.11-1.41$ & $<0.01$ \\
\hline High & 1.92 & $1.71-2.16$ & $<0.01$ \\
\hline Unknown & 1.32 & $1.15-1.5$ & $<0.01$ \\
\hline \multicolumn{4}{|l|}{ Size $(\mathrm{cm})$} \\
\hline$<2$ & Reference & & \\
\hline $2-5$ & 1.54 & $1.42-1.68$ & $<0.01$ \\
\hline$>5$ & 2.43 & $2.16-2.75$ & $<0.01$ \\
\hline Unknown & 1.01 & $0.94-1.09$ & 0.50 \\
\hline \multicolumn{4}{|l|}{ ER } \\
\hline Positive & Reference & & \\
\hline Equivocal & 1.21 & $0.49-3.31$ & 0.66 \\
\hline
\end{tabular}




\begin{tabular}{llll}
\hline & OR & $\mathbf{9 5} \% \mathbf{C I}$ & $\boldsymbol{P}$ \\
\hline Negative & 1.05 & $0.9-1.22$ & 0.46 \\
Unknown & 0.37 & $0.3-0.45$ & $<0.01$ \\
Prior non-breast malignancy & & & \\
No & Reference & & \\
Yes & 0.57 & $0.53-0.61$ & $<0.01$ \\
\hline
\end{tabular}

$E R$ estrogen receptor; $O R$ odds ratio; $C I$ confidence interval 


\section{TABLE 3}

Patient and tumor characteristics associated with the odds of receiving a sentinel lymph node biopsy among patients for whom DCIS represented the one and only known malignancy $(N=14,699)$

\begin{tabular}{|c|c|c|c|c|}
\hline & SLNB $N=7,882(\%)$ & OR & $95 \% \mathrm{CI}$ & $P$ \\
\hline \multicolumn{5}{|l|}{ Age (years) } \\
\hline$\leq 6$ & $4,717(60)$ & Reference & & \\
\hline$>56$ & $3,164(40)$ & 0.64 & $0.60-0.69$ & $<0.01$ \\
\hline \multicolumn{5}{|l|}{ Year } \\
\hline 2000 & $292(4)$ & Reference & & \\
\hline 2001 & $443(6)$ & 1.48 & $1.24-1.76$ & $<0.01$ \\
\hline 2002 & $596(8)$ & 2.04 & $1.72-2.41$ & $<0.01$ \\
\hline 2003 & $698(9)$ & 3.08 & $2.61-3.65$ & $<0.01$ \\
\hline 2004 & $868(11)$ & 2.92 & $2.44-3.48$ & $<0.01$ \\
\hline 2005 & $1,006(13)$ & 4.12 & $3.43-4.96$ & $<0.01$ \\
\hline 2006 & $1,198(15)$ & 5.29 & $4.40-6.34$ & $<0.01$ \\
\hline 2007 & $1,262(16)$ & 5.08 & $4.22-6.13$ & $<0.01$ \\
\hline 2008 & $1,519(19)$ & 7.52 & $6.22-9.10$ & $<0.01$ \\
\hline \multicolumn{5}{|l|}{ SEER registry } \\
\hline West & $4,601(58)$ & Reference & & \\
\hline East & $1,287(16)$ & 1.4 & $1.26-1.56$ & $<0.01$ \\
\hline Midwest & $1,302(17)$ & 1.02 & $0.91-1.13$ & 0.77 \\
\hline South & $692(9)$ & 1.07 & $0.93-1.22$ & 0.33 \\
\hline \multicolumn{5}{|l|}{ Race } \\
\hline White & $5,759(73)$ & Reference & & \\
\hline Asian or PI & $854(11)$ & 0.78 & $0.70-0.88$ & $<0.01$ \\
\hline Black & $667(9)$ & 0.91 & $0.80-1.04$ & 0.18 \\
\hline Hispanic & $513(7)$ & 0.86 & $0.74-1.00$ & 0.05 \\
\hline Native American/Alaskan & $37(<1)$ & 1 & $0.59-1.70$ & 0.99 \\
\hline Unknown & $53(1)$ & 0.81 & $0.53-1.25$ & 0.34 \\
\hline \multicolumn{5}{|l|}{ Grade } \\
\hline Low & $474(6)$ & Reference & & \\
\hline Intermediate & $2,158(27)$ & 1.27 & $1.10-1.47$ & $<0.01$ \\
\hline High & $4,236(54)$ & 1.9 & $1.64-2.20$ & $<0.01$ \\
\hline Unknown & $1,014(13)$ & 1.34 & $1.14-1.57$ & $<0.01$ \\
\hline \multicolumn{5}{|l|}{ Size $(\mathrm{cm})$} \\
\hline$<2$ & $2,627(33)$ & Reference & & \\
\hline $2-5$ & $1,910(24)$ & 1.53 & $1.38-1.68$ & $<0.01$ \\
\hline$>5$ & $1,055(13)$ & 2.41 & $2.10-2.77$ & $<0.01$ \\
\hline Unknown & $2,290(29)$ & 1.03 & $0.94-1.12$ & 0.5 \\
\hline \multicolumn{5}{|l|}{ ER } \\
\hline Positive & $3,522(45)$ & Reference & & \\
\hline Equivocal & $15(<1)$ & 1.38 & $0.46-4.83$ & 0.58 \\
\hline
\end{tabular}




\begin{tabular}{lllrr}
\hline & SLNB $N=\mathbf{7 , 8 8 2}(\boldsymbol{\%})$ & OR & $\mathbf{9 5} \% \mathbf{C I}$ & $\boldsymbol{P}$ \\
\hline Negative & $1,123(14)$ & 1.03 & $0.86-1.25$ & 0.74 \\
Unknown & $3,222(41)$ & 0.37 & $0.28-0.47$ & $<0.01$ \\
\hline
\end{tabular}

$S L N B$ sentinel lymph node biopsy, $E R$ estrogen receptor, $D C I S$ ductal carcinoma in situ, $O R$ odds ratio, $C I$ confidence interval 